\title{
Thermoresponsive hydrogels for atmospheric water vapor harvesting
}

Boyuan $\mathrm{Hao}^{1,{ }^{*}}$, Pavani Cherukupally ${ }^{1, *}$, Zhongshen Zhang $^{2}$, Daryl Williams ${ }^{1}$

${ }^{1}$ Surface Particle Engineering Laboratory, The Department of Chemistry, Imperial College London, London, SW7 2AZ, UK.

${ }^{2}$ National Engineering Laboratory for VOCs Pollution Control Material \& Technology, University of Chinese Academy of Sciences, 100096, China.

*These authors contributed equally

\begin{abstract}
Currently, freshwater scarcity is a global challenge that is threatening four billion people across the world. To satisfy people's increasing freshwater demand, harvesting atmospheric water from the air could be an alternative way. This work developed copolymer P(NIPAM-co-15\%BzDMA) hydrogels to harvest atmospheric water vapor. Two methods were investigated to improve its adsorption performance: decreasing synthesis temperature below the LCST and copolymerizing with the optimum amount of quaternary ammonium salt (QAS). We found these two methods can effectively improve the water vapor uptake. After copolymerizing NIPAM with $15 \%$ QAS at $20^{\circ} \mathrm{C}$, the water vapor uptake could be increased by almost $20 \%$ to $232 \mathrm{mg} / \mathrm{g}$ at $20^{\circ} \mathrm{C}$ and $\mathrm{P} / \mathrm{P} 0$ of 0.75 compared with pure PNIPAM prepared at $60^{\circ} \mathrm{C}(194 \mathrm{mg} / \mathrm{g})$. The significant increase can be attributed to the more uniform porous structure and the hygroscopicity of QAS. After coating PNIPAM onto the PESPU sponge skeleton, the PESPU-PNIPAM_60 could adsorb 180 mg/g gas water at $20^{\circ} \mathrm{C}$ and $\mathrm{P} / \mathrm{P} 0$ of 0.75 , and the modified sponges inherit the switchable wettability from PNIPAM. This research provides polymer processing parameters and their character for harvesting water vapor from the air with hydrogels.
\end{abstract}

Keywords: hydrogel; thermoresponsive polymers, moisture-responsive polymers, water vapor harvesting 


\section{Introduction}

Although, $0.26 \%$ of freshwater reserves are accessible, about 4 billion people in the world suffer from water scarcity $(1,2)$. Alternatively, the 13000 trillion liters of water in the air, in the form of vapor and droplets, could address water scarcity challenges, especially in arid regions (3). To date, three strategies to capture water from the air have been reported: (a) fog collection on cooling nets, (b) direct cooling air below the dew point, and (c) water vapor sorption by adsorbents (4). Due to the minimum requirement of the relative humidity $(\mathrm{RH})$ above $50 \%$, the usage of the fog collectors are limited to heavy fog containing regions of the world (5). Whereas the low partial pressure of water vapor at ambient temperatures makes its cooling below dew point temperature energy intensive process. Alternatively, the partial pressure of water vapor released from the adsorbent is increased compared to that of water vapor in moist air, which is much easier to reach the saturated vapor pressure required for condensation. Using this strategy, water vapor can be captured using adsorbents at nights when the RH is high, and released using sunlight during the day (5).

Recently, porous materials, such as metal organic frameworks (MOFs), hydrogels, and polymeric sponges have emerged as the promising adsorbents for enhanced water harvesting across all climate conditions $(4,6,7,8,9)$. Due to their large surface area-to-volume ratio, the porous materials could adsorb, store, and transmit larger amount water molecules at a shorter timescale. MOFs usually exhibit S-shaped adsorption-desorption isotherms meaning captured water can be collected without losses (6). However, they suffer from long-term hydrolytic and architectural instabilities $(3,4)$. Furthermore, the large-scale manufacturing of MOFs that can retain captured water is still a big challenge (4). Alternatively, thermoresponsive polymeric hydrogels, such as polypyrrole chloride penetrated PNIPAM hydrogel, can capturing moisture at night at low temperatures and recover in the morning using sunlight (10). Another approach is to apply superhydrophilic polymeric sponges for water harvesting. The sponges fabricated with cellulose fibers, and functionalized with graphite, poly (sodium acrylate), could capture water by adsorption and release by mechanical compression or heating with sunlight $(7,9)$. Due to low cost, tunable surface properties, excellent mechanical compression, the polymeric sponges could become affordable water harvesters for practical implementation. The performance of the sponges could be further enhanced by enhancing their surface properties with thermoresponsive hydrogels. 
This work develops a new approach to enable superhydrophilic-superhydrophobic sponges for water harvesting. This adsorption-desorption is driven by the hydrophilic-hydrophobic properties of the thermoresponsive, nanoporous hydrogel coupled with microporous, polymeric sponges. At below $32{ }^{\circ} \mathrm{C}$, commonly seen at night, due to superhydrophilic properties, the hydrogels, such as PNIPAM, could capture water vapor from ambient air and swell. While above $32{ }^{\circ} \mathrm{C}$, commonly seen during day, due to hydrophobic properties, the captured water vapor will be released. The groovy topography could enhance condensation of the captured water vapor for energy-efficient release $(11,12)$. The high compressive strength of the flexible sponge also enables water release by passive compression. Furthermore, the hierarchical porosity of the composite increases the net surface area-to-volume ratios for collecting larger volume of water vapor.

Herein, a nanoporous hydrogels with P(NIPAM-co-BzDMA) for passive water harvesting from air. The P(NIPAM-co-BzDMA) was developed by copolymerizing the monomeric NIPAM (NIsopropylacrylamide) with BzDMA (N-benzyl-2-(methacryloyloxy)-N, N-dimethylethanaminium chloride), and free-drying to increase its porosity and mechanical stability. The hydrogels were covalently attached to the PU surfaces pretreated with ViTS (Vinyltrichlorosilane) for good adhesion. The chemical characterization, optical, and electron micrographs, show the hydrogels exhibit thermoresponsive superhydrophilic-superhydrophobic properties and hierarchical porosity. The static and dynamic water vapor adsorption tests show the hydrogels could uptake water vapor at 0 to $90 \%$ and $20{ }^{\circ} \mathrm{C}$. Based on these results, the P(NIPAM-co-BzDMA) sponges could serve as the affordable, ultrafast water harvesters across all environmental conditions.

\section{Experimental}

2.1. Materials: A commercial PU sponge was purchased from a local vendor. The $\mathrm{N}$ isopropylacrylamide (NIPAM) monomer, methylene-bis-acrylamide (MBA) crosslinker, Vinyltrichlorosilane (ViTS) binder, and n-hexane, acetone, ethanol solvents were purchased from Sigma Aldrich. N-benzyl-2-(methacryloyloxy)-N, N-dimethylethanaminium chloride (ca.75\% in water) stabilized with MEHQ (BzDMA) were purchased from TCI. The initiators, potassium persulfate (KPS) and N, N, N', N'-tetramethylethylenediamine (TEMED) were purchased from MACKLIN. 


\subsection{Materials fabrication}

PNIPAM synthesis: The synthesis procedure was modified from previous reports $(13,14)$. PNIPAM switches from hydrophobic to hydrophilic at its lower critical solution temperature (LCST) of $\leq 32^{\circ} \mathrm{C}(15)$. The change in polymeric structure with temperature could influence its efficacy of crosslinking. Therefore, the PNIPAM was synthesized above and below LCST that is at 60 and $20^{\circ} \mathrm{C}$. NIPAM (7.7 g), MBA (0.2 g), KPS (0.05 g), and TEMED (43 $\left.\mu \mathrm{L}\right)$ were added to deionized water ( $77 \mathrm{~g}$ ) in sequence, and then magnetically stirred at 60 or $20^{\circ} \mathrm{C}$ for $4 \mathrm{hrs}$ under the nitrogen atmosphere. Then, the PNIPAM was washed several times with deionized water in an ultrasonic bath. The cleaned PNIPAM was freeze-dried at approximately $-40{ }^{\circ} \mathrm{C}$ and $20 \mathrm{~Pa}$ in vacuum for 12 hrs. After freeze-drying, the target product PNIPAM@60 and PNIPAM@20 hydrogels were obtained. The results (see Results section 3.3) indicated that PNIPAM@20 exhibited relatively higher water uptake due to more uniform porous structure with relatively larger surface area hence subsequent material synthesis was performed at this temperature.

Copolymer aerogels synthesis: The P(NIPAM-co-BzDMA) was synthesized at $20^{\circ} \mathrm{C}$, where the NIPAM could copolymerize with BzDMA efficiently. The copolymeric solution was prepared by adding the mixture of NIPAM, MBA, KPS, and BzDMA at concentrations 10, 15, and 20 wt $\%$ (Table S1) to $77 \mathrm{~g}$ of deionized water. The solution was magnetically stirred at approximately 20 ${ }^{\circ} \mathrm{C}$ under the nitrogen atmosphere. After $15 \mathrm{~min}$, TEMED initiator was added to the mixing solution and the reaction was continued for $4 \mathrm{hrs}$ to produce the gel. The gel was washed several times with deionized water in an ultrasonic bath and freeze dried at approximately $-40{ }^{\circ} \mathrm{C}$ and 20 $\mathrm{Pa}$ in vacuum for $12 \mathrm{hrs}$. The freeze drying produced nanopores in the copolymers via ice sublimation. The resulting porous copolymer is referred to as a aerogel from here onwards. The freeze-dried copolymers with increasing contents of quaternary ammonium salts (QAS), referred to as P(NIPAM-co-10\%BzDMA), P(NIPAM-co-15\%BzDMA), and P(NIPAM-co-20\%BzDMA), were evaluated by static adsorption test to select the best candidate for coating the PU sponges (see Results section 3.3). The sorption tests suggested among three concentrations, the 15\%BzDMA produced higher water uptakes. 


\subsection{Materials Characterization}

Surface properties: Change in functional groups of the materials was evaluated using Fourier transform infrared spectroscopy (FT-IR). The analysis of the samples was carried out on a Tensor 27 infrared spectrometer (Bruker, Germany) in the absorption range of 850 to $4000 \mathrm{~cm}^{-1}$ at a resolution of $1 \mathrm{~cm}^{-1}$. The surface elemental composition of the samples was determined using $\mathrm{X}$ ray photoelectron spectroscopy (XPS, Thermo Fisher Scientific, ESCALAB 250Xi, USA). Al Ka $\mathrm{X}$-ray radiation was used as the $\mathrm{X}$-ray source for excitation and the beam spot was $650 \mu \mathrm{m}$. The spectra were obtained using of a pass energy of $20 \mathrm{eV}$ with an instrument resolution of $0.050 \mathrm{eV}$. The surface morphology and crosslinking structure of the PNIPAM-based samples were probed on a scanning electron microscopy (SEM). All SEM images were collected by a Hitachi instrument (su8020, Japan). The SEM images were obtained at different magnifications at an accelerating voltage of $15 \mathrm{kV}$. The phase change of materials could be detected on Differential scanning calorimetry (DSC250, TA instrument) and the LCST could be determined. The dry samples were immersed in deionized water at room temperature and was moved out after $24 \mathrm{hrs}$; excess water in the samples could be sucked out with a filter; 3-5 mg of the wet sample in a closed pan was heated at a heating rate of $10^{\circ} \mathrm{C} / \mathrm{min}$ from $10^{\circ} \mathrm{C}$ to $60^{\circ} \mathrm{C}$ and conditioned under a nitrogen purge of $20 \mathrm{~mL} / \mathrm{min}$. The heat flow between the sample and the reference was recorded. Water contact angles (WCA) of the modified sponges were measured using a drop shape analyzer (Tracker, France Teclis) at 25 and $40{ }^{\circ} \mathrm{C}$. Prior to measurements, the sponge sample and the water were preheated or cooled to desired temperatures, then the drop was placed on the sponge surface. With the help of instruments software, the images were captured and the WCA were measured over a period.

Ellipsometry and DVS: The efficacy of the coating concentration for maximum amount of water vapor sorption was evaluated using an ellipsometry. The dynamic adsorption and desorption of water vapor and coatings swelling measurements were performed using a Dynamic Vapor Sorption (DVS) instrument (Surface Measurement Systems Ltd., UK) coupled with a FS-1 multiwavelength ellipsometer (Film Sense, USA). The water vapor sorption experiments were carried out in an environmental chamber at a temperature of $20{ }^{\circ} \mathrm{C}$, at atmospheric pressure and in a relative humidity range between 0 and $80 \%$. The relative humidity was maintained using mass- 
flow controllers operating in open-loop mode using dry air as the carrier gas with a flowrate of $200 \mathrm{~mL} \mathrm{~min}^{-1}$ and monitored using a humidity probe. The ellipsometer uses four wavelengths (465, 525,595 and $635 \mathrm{~nm}$ ) at a chosen optimal angle of incidence between $65^{\circ}$ and $70^{\circ}$, whichever produced the highest possible signal intensity. The as-prepared samples of films and layers were measured in parallel and equilibrated at each relative humidity level in steps of $5 \%$ to produce isotherms. The DVS was used to monitor the change in mass of layered sample using a microbalance (resolution of $0.1 \mu \mathrm{g}$ ) and the ellipsometer to monitor the change in film thickness and optical properties of PNIPAM or copolymer films.

Static adsorption/desorption study of water vapor: The adsorption and desorption of water vapor onto the PNIPAM-based sponges under static conditions were evaluated using an intelligent gravimetric analyzer (IGA-100, supplied by Hiden Isochema Ltd., Warrington, U.K.). The analyzer was equipped with an ultrahigh vacuum (UHV) system, a high-precision microbalance, a temperature, and pressure control module, which are controlled by the instrument's software. The microbalance used to measure change in sample weights has a stability of $\pm 1 \mu \mathrm{g}$, a resolution of $0.2 \mu \mathrm{g}$, and can weight samples up to $200 \mathrm{mg}$. Approximately $60 \mathrm{mg}$ of the sponge sample was used for each measurement. Prior to measurements, the sample was degassed at $90{ }^{\circ} \mathrm{C}$ for 5 hrs at a pressure $<10^{-6} \mathrm{~Pa}(16)$.

Deionized water was used as a source to generate water vapor at $55^{\circ} \mathrm{C}$, and the gas circuit was cleaned by repeated evacuation and vapor generation equilibration cycles. Fully computerized UHV system and high-precision pressure control valve allowed adsorption isotherms to be obtained by setting pressure steps (16). The pressure steps were set depending on the saturated vapor pressure of water at the temperature used for the adsorption measurement at the highest saturated vapor pressure (i. e. relative humidity) of $80 \%$. The saturated vapor pressures of water at $20{ }^{\circ} \mathrm{C}$ was 23.88 mbar. Therefore, the highest pressures were set as 19 and 59 mbar, respectively. The accuracy of the set pressure point could reach $0.02 \%$ for the employed range. During the experiments, the set pressure value was automatically controlled by a UHV system and a highprecision pressure control valve. For each pressure point, the minimum and maximum times for adsorption equilibrium were 30 and $60 \mathrm{~min}$, respectively. The temperature of the sample was 
recorded using a thermocouple placed $5 \mathrm{~mm}$ from the sample. The saturated vapor pressures were calculated using Antoine equation,

$$
\log p_{o}=A-\frac{B}{T+C}
$$

where $p_{o}$ is the saturated vapor pressure (mbar) corresponding to the temperature $\mathrm{T}\left({ }^{\circ} \mathrm{C}\right)$, and $\mathrm{A}$, $\mathrm{B}$ and $\mathrm{C}$ are adsorbate dependent constants. The A, Band C parameters used for water are 8.0955, 1747.3 and 235.07, respectively (17). The adsorption and desorption isotherms of the samples were obtained at 20 and $40^{\circ} \mathrm{C}$. The adsorption quantity of an adsorbent could be calculated gravimetrically according to,

$$
q=\frac{m_{w}-m_{d}}{m_{d}} \times 100
$$

where $m_{d}$ represents the initial weight of the dry sample $(\mathrm{mg})$ and $m_{w}$ is the total weight of the wet sample (mg).

Scalability study at variable RH: For scalability, approximately $1 \mathrm{~g}$ of the sponge samples were used to adsorb the water vapor at RH values of 20 to $80 \%$ at $20 \%$ intervals. The desired RH value was achieved with the help of humidity generator connected to a desiccator at room temperature. Each adsorption experiments were performed for $24 \mathrm{hrs}$ where change in sample weights were measured gravimetrically at predefined intervals using equation 2 . The adsorbed water from the sponges were collected either using suction or mechanical compression.

Sponge fabrication: The hydrogel coated sponges were modified based on the method reported previously $(13,14)$. Prior to usage, approximately $2 \times 2 \times 2 \mathrm{~cm}^{3}$ of sponges were cleaned with acetone, deionized water, and ethanol in an ultrasonic bath and drying at $60{ }^{\circ} \mathrm{C}$ for $1.5 \mathrm{hrs}$. The sponge samples were suspended in hexane $(40 \mathrm{~mL})$ and ViTS $(200 \mu \mathrm{L})$ mixture for 30 minutes, then dried at $100{ }^{\circ} \mathrm{C}$ for $2 \mathrm{hrs}$ to obtain the silanized PU-ViTS. The ViTS acts as a binder between the PU sponge and the hydrogel in enhancing the mechanical stability of coating. Hydrogel solution at 15\%BzDMA (Table S1) and silanized PU-ViTS were magnetically stirred in deionized water for 4 hrs under nitrogen atmosphere to produce hydrogel coated sponges, referred to as composites from here onwards. The composites were washed several times with deionized water 
in an ultrasonic bath. The target products PU-P(NIPAM-co-15\%BzDMA) were obtained after freeze drying at approximately $-40{ }^{\circ} \mathrm{C}$ and $20 \mathrm{~Pa}$ in vacuum for $12 \mathrm{hrs}$.

\section{Results and Discussions}

\subsection{Aerogels synthesis and chemical structure}
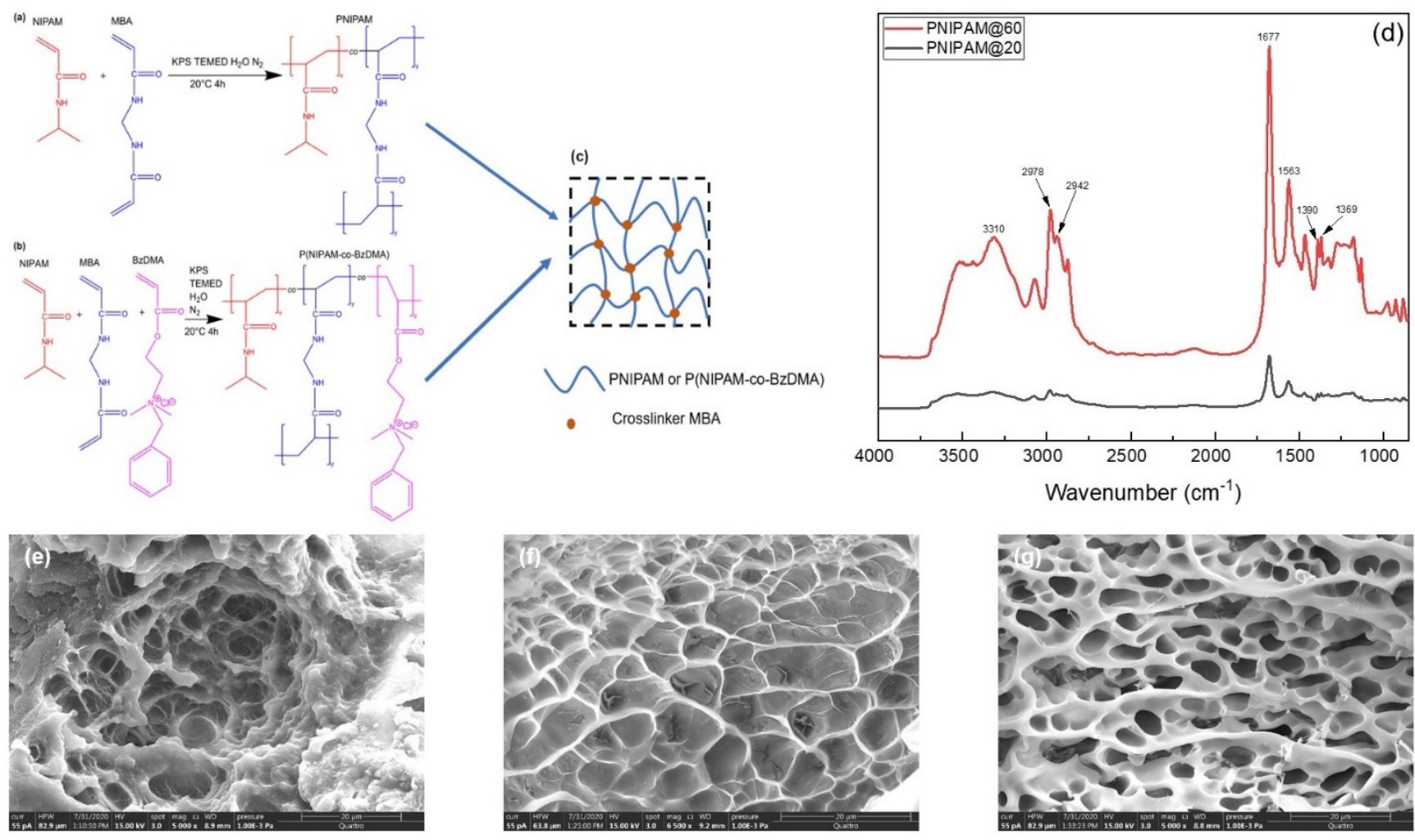

Figure 3.1 Representation of free radical polymerization of (a) PNIPAM, (b) P(NIPAM-coBzDMA) and (c) the crosslinking network of the prepared hydrogel, (d) FT-IR spectra of PNIPAM synthesized at different temperatures, SEM images of (e) PNIPAM@60, (f) PNIPAM@20, (g) P(NIPAM-co-10\%BzDMA) at different magnification.

In this research, we synthesized pure PNIPAM (Figure 3.1 (a)) and its copolymers with quaternary ammonium salt P(NIPAM-co-BzDMA) (Figure 3.1 (b)) by thermally initiated polymerization. The KPS and TEMED were used as initiators to generate more free radicals. After polymerization, monomeric NIPAM itself or NIPAM and BzDMA can be crosslinked by the crosslinker MBA to form a network structure (Figure 3.1 (c)). 
Chemical structure: The formation of PNIPAM prepared at different synthesis temperatures can be confirmed by FT-IR spectra presented in Figure 3.1(d).PNIPAM@60 and PNIPAM@20 both show a typical FT-IR spectrum of PNIPAM. The most intense peak at $1677 \mathrm{~cm}^{-1}$ is attributed to $\mathrm{C}=\mathrm{O}$ and the second most intense peak at $1563 \mathrm{~cm}^{-1}$ is due to amide groups $(\mathrm{N}-\mathrm{H}$ and $\mathrm{C}-\mathrm{N})$; characteristic bimodal peaks of isopropyl groups could be observed at 1390, and $1379 \mathrm{~cm}^{-1}$; methylene peaks could be seen at 2987 and $2942 \mathrm{~cm}^{-1}(18)$; the broad peak at $3310 \mathrm{~cm}^{-1}$ is caused by N-H stretching (19). However, the peaks of PNIPAM@60 are much more intensive than those of PNIPAM@20 because heating at higher temperature provides more oxygen-involved radicals to readily crosslink NIPAM.

Morphology of the aerogels: The morphology and porosity of PNIPAM and copolymers $\mathrm{P}$ (NIPAM-co-BzDMA) are observed by SEM. The aerogels present interconnected microporous structures. The formation of the amorphous structure is caused by the freeze drying. (20) Compared with PNIPAM@60 (Figure 3.1 (e)), PNIPAM@20 (Figure 3.1 (f)) has relatively more evenly distributed pores which are suitable for water vapor to transfer inside. The BET results also show that the surface area of PNIPAM@20 $\left(2.91 \mathrm{~m}^{2} / \mathrm{g}\right)$ is slightly larger than that of PNIPAM@60 $\left(2.67 \mathrm{~m}^{2} / \mathrm{g}\right)$. Therefore, the synthesis temperature has a significant influence on the porous structure of the resultant polymer. When polymerization is conducted below the LCST, the reaction rate is relatively slow, and the whole reaction system is always in the homogenous phase during the polymerization, which can make the porous structure of the sample more uniform. However, above the LCST, polymerization occurs very fast, and the NIPAM in the mixed solution excludes water because of its hydrophobicity which makes the reaction system heterogeneous, therefore producing nonuniform pores. After NIPAM is copolymerized with BzDMA, the copolymers (Figure 3.1(g)) can still maintain an excellent porous structure.

\subsection{Sponge fabrication and switchable wettability}

Here, the synthesis route for a 3D PU sponge skeleton with temperature-controlled wettability for water vapour capture from air is shown in Figure 3.2(a). First, the PU sponge is used as a 3D substrate with a uniform porous structure. Then, hydrolysation and polycondensation of vinyl trichlorosilane (ViTS) on the surface of the PU sponge can provide vinyl groups. Finally, NIPAM 
is polymerized, or NIPAM and BzDMA are copolymerized in the pores of the sponge. At the same time, PNIPAM or P(NIPAM-co-BzDMA) can also be interlinked by ViTS onto the PU skeleton.

(a)

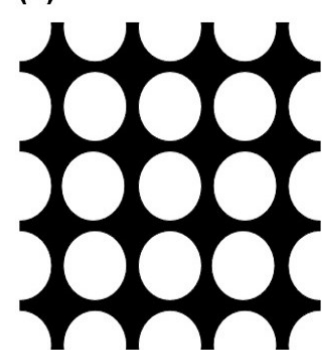

Cleaned PESPU

\section{Silanization}
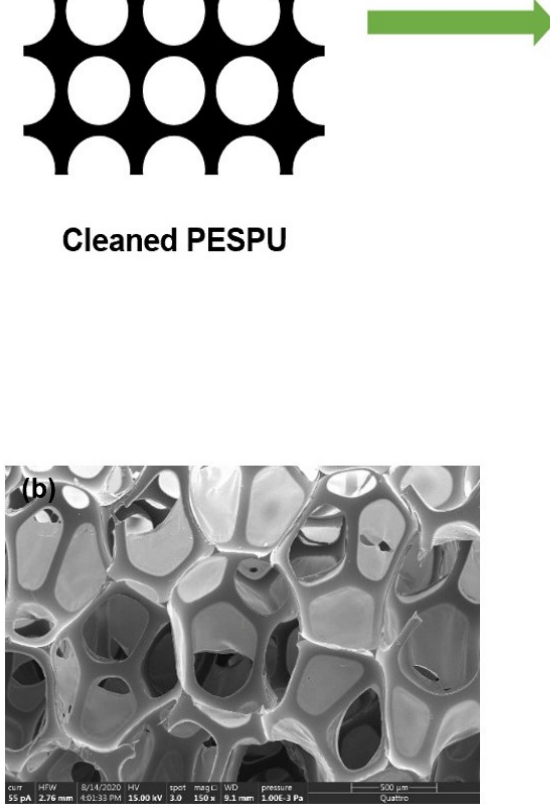

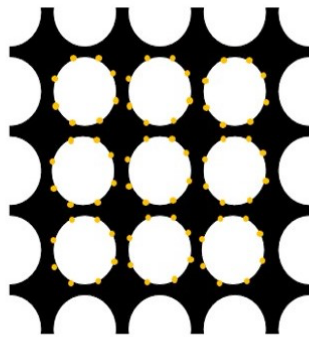

PU-ViTS

Inter-linker ViTS

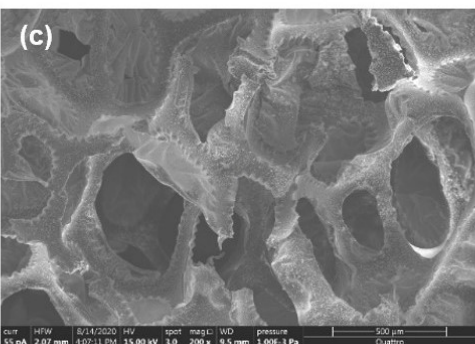

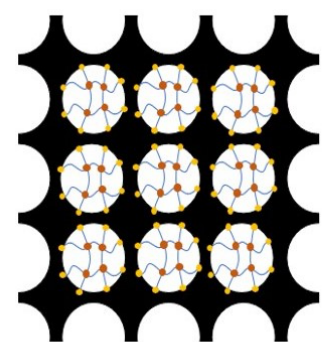

PU-P(NIPAM-co-BzDMA)

PNIPAM or P(NIPAM-co-BzDMA)

Crosslinker MBA

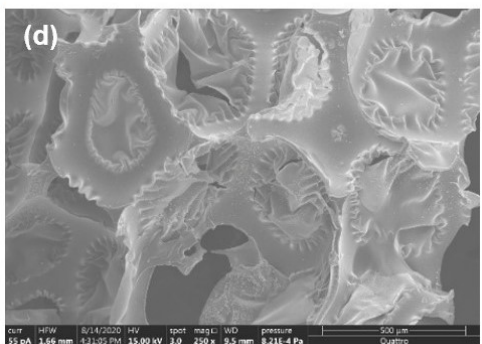

Figure 3.2 (a) Synthesis route for sponge fabrication, SEM images of (b) cleaned PU, (c) PUViTS, (d) PU-P(NIPAM-co-20\%BzDMA).

Morphology of fabricated sponges: The sponge fabrication processes could be detected by SEM images which show the microstructure change in the pores of the PU sponge during modification. At the beginning, the pores of the PU sponge look smooth and clean (Figure 3.2(b)). After silanization, the surface of PU-ViTS is covered with some particles that are formed by the hydrolytic polycondensation of ViTS on the surface of sponge skeleton (Figure 3.2(c)). After modification with the copolymer, some structures can be observed in the initially empty pores and adhere to the sponge skeleton because the NIPAM and BzDMA crosslink with each other to form aerogel and simultaneously the aerogel interlinks with the surface of the sponge skeleton (Figure 
3.2(d)). The sequent SEM images implies that this synthesis method can successfully coat aerogel onto the sponge.
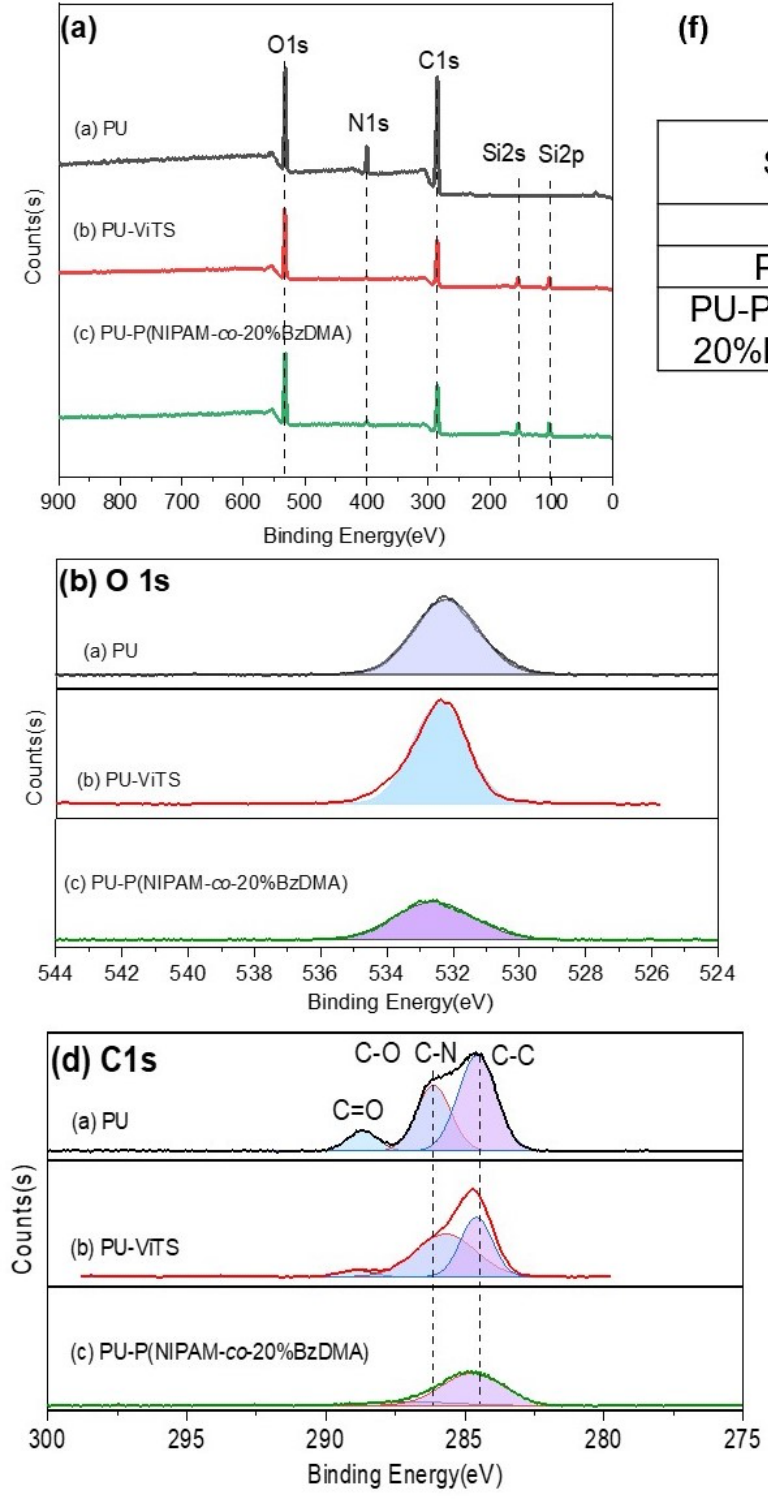

(f)

\begin{tabular}{|c|c|c|c|c|}
\hline \multirow{2}{*}{ Sample } & \multicolumn{4}{|c|}{ Atomic content (\%) } \\
\cline { 2 - 5 } & Si 2p & C 1s & N 1s & O 1s \\
\hline PU & 0 & 69.43 & 8.11 & 22.12 \\
\hline PU-ViTS & 12.99 & 55.65 & 2.42 & 28.94 \\
\hline $\begin{array}{c}\text { PU-P(NIPAM-co- } \\
\text { 20\%BzDMA) 20 }\end{array}$ & 14.21 & 53.70 & 3.03 & 29.06 \\
\hline
\end{tabular}
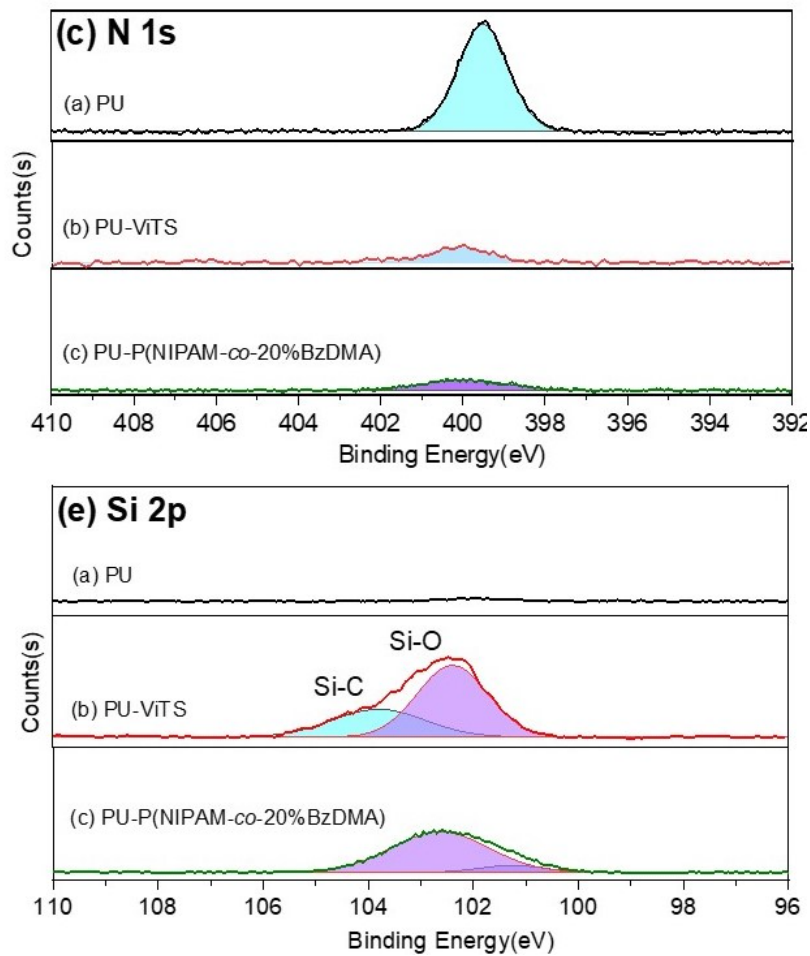

Figure 3.3 XPS spectra of (a) the full, (b) O 1s, (c) N 1s, (d) C 1s, and (e) Si 2p regions of the fabricated sponges, (f) Elemental composition analysis of sponge surfaces based on XPS spectra.

Surface properties: The surface elemental composition of the sponge could be measured by XPS spectroscopy analysis as shown in Figure 3.3(a) of the full. The spectra of O 1s and N 1s (Figure 3.3(b) and (c)) displayed that the peaks located at $532.3 \mathrm{eV}$ and $400.2 \mathrm{eV}$ originated from the presence of chemical oxygen species and nitrogen species, respectively. The spectra of $\mathrm{C} 1 \mathrm{~s}$ 
(Figure 3.3(d)) displayed three species: the peak located at $288.8 \mathrm{eV}$ is assigned to surface $\mathrm{C}=\mathrm{O}$, the peak centred at $286.3 \mathrm{eV}$ originated from the presence of $\mathrm{C}-\mathrm{O}$ or $\mathrm{C}-\mathrm{N}$, and the peak located at $284.6 \mathrm{eV}$ corresponding to surface C-C. The peaks located at $104.2 \mathrm{eV}$ and $102.5 \mathrm{eV}$ are due to surface Si-C and Si-O, respectively (Figure 3.3(e)). (14)

The atomic contents of $\mathrm{Si}, \mathrm{C}, \mathrm{N}, \mathrm{O}$ for each sample could be calculated which are presented in Figure 3.3(f). Compared with PU, PU-ViTS contains Si, and the content of O is increased, because hydrolysis and polymerization occur on the sponge surface to form a polysiloxane layer. However, the atomic content of $\mathrm{N}$ in PU-ViTS decreases, which could be attributed to the shielding effect of the silicone layer on the sponge surface. After modification, a slight increase in nitrogen content could be observed in PU-P(NIPAM-co-20\%BzDMA), compared with PU-ViTS, because PNIPAM and BzDMA contain more N element. (14)

Switchable wettability of fabricated sponges: The water contact angles (WCAs) of PUP(NIPAM-co-20\%BzDMA) were tested under different temperatures of 25 and $40^{\circ} \mathrm{C}$, and the detailed results are presented in Figure 3.4(a)-(b). The modified sponge is superhydrophilic with a WCA of $0^{\circ}$ when the temperature is below the LCST. The water droplet can penetrate through the pores of PU-P(NIPAM-co-20\%BzDMA) sponge within 40 seconds. When the ambient temperature increases above the LCST, PU-P(NIPAM-co-20\%BzDMA) sponge turns to be hydrophobic, with the average WCAs of approximately $93.6^{\circ}$ within 540 seconds. According to Yi's results ((14), after modifying PU sponge with the coupling agent ViTS, the silanized sponge exhibits superhydrophobicity, with a WCA of $158^{\circ}$ because of the nanoscale roughness and hydrophobic silicone particles on the surface of the sponge, which will not change with temperature. Compared with PU-ViTS, PU-P(NIPAM-co-20\%BzDMA) exhibit a sensitive temperature response and excellent tunable wettability because of the nature of PNIPAM.

\subsection{Static adsorption and desorption test}

Water vapor adsorption/desorption isotherms of aerogels were measured on an IGA at 20 and $40^{\circ} \mathrm{C}$ and were gravimetrically obtained as shown in Figure S2 and S3. These isotherm shapes more 
closely resemble type II than other types, which indicates the existence of macropores in the materials as shown in the SEM images in 3.1. For PNIPAM@60 (Figure S2(a)-(b)) and PNIPAM@20 (Figure S2 (c)-(d)), the water vapor uptake increases linearly as a function of relative pressure. For the copolymers P(NIPAM-co-10\%BzDMA) (Figure S3 (a)-(b)), P(NIPAMco-15\%BzDMA) (Figure 3.4 (c)-(d)) and P(NIPAM-co-20\%BzDMA) (Figure S3(c)-(d)), the adsorption branch shows a convex shape at lower relative pressures followed by a concave shape at higher relative pressures. According to Smith's theory, there are two principal stages of adsorbed water corresponding to the two regions in the adsorption branch. At the first stage, water molecules are condensed within the polymeric adsorbent as a function of relative pressure (corresponding to the convex shape). At the second stage, the water molecules are bound to the inner or outer surface of polymeric adsorbent (corresponding to the concave shape) $(24,25)$.

The area of the hysteresis loops can be utilized to reflect the wettability of the material. The larger hysteresis loop obtained at $20^{\circ} \mathrm{C}$ is larger than that obtained at $40{ }^{\circ} \mathrm{C}$. For example, for pure PNIPAM@60, it is evident that the hysteresis loop of the adsorption/desorption isotherm obtained at $20^{\circ} \mathrm{C}$ is slightly larger than that obtained at $40^{\circ} \mathrm{C}$, because PNIPAM is hydrophilic under the LCST, and thus, the adsorbed water has difficulty escaping the adsorbent due to the strong interaction between water molecules and the hydrophilic groups of PNIPAM. In contrast, when the temperature is above the LCST, the area of the hysteresis loop decreases. The switchable hydrophilic-hydrophobic property of PNIPAM could be determined by DSC thermograms as shown in Figure 3.4 (e). This phenomenon can also be observed on the three copolymers. Significantly, the three copolymers do not display a hysteresis loop when the relative pressure is above 0.65 which implies that they are superhydrophobic in this region, making the water escape the adsorbent easily.

The adsorption capacities of PNIPAM and the copolymers $\mathrm{P}\left(\mathrm{NIPAM}-\right.$ co-BzDMA) at $\mathrm{P} / \mathrm{P}_{0}=0.75$ can be obtained from the isotherms which were summarized and listed in Table 3.2. From these results, the influence of some different factors on water vapor uptake could be explored.

First, the effect of environment temperature $\left(20\right.$ and $\left.40^{\circ} \mathrm{C}\right)$ was investigated. Generally, for chemisorption which requires activation energy, an increase in temperature can provide heat to 
activate gas molecules and therefore promote more gas to be adsorbed. We found that the adsorption capacity below the LCST is higher than that above the LCST for all of the samples except for the copolymer with high content of BzDMA P(NIPAM-co-20\%BzDMA), which show an increase in adsorption capacity with the increase in temperature from 20 to $40^{\circ} \mathrm{C}$. Therefore, the decrease in adsorption capacity with increasing temperature for most samples can be attributed to the balanced effect of chemisorption and switchable wettability. In addition, it must be pointing out that although those PNIPAM-based polymers show limited tunable wettability to gas water, they are not as sensitive as to liquid water, which could be seen from the small difference in the adsorption capacity of water vapor between $20^{\circ} \mathrm{C}$ and $40^{\circ} \mathrm{C}$. After all, it is more difficult for PNIPAM to change its configuration of chains in a gaseous environment than in a liquid environment to switch its wettability.

Second, the synthesis temperature also has a significant effect on the adsorption capacity. PNIPAM @20 exhibits a higher water vapor uptake than PNIPAM@60. When polymerization is conducted below the LCST, the whole system is in a homogenous phase, and therefore forming more evenly distributed pores, which contribute to adsorption. This result is in good agreement with the SEM images shown in Figure 3.1(e)-(g).

Third, the addition of QAS can also affect the adsorption of water vapor. As shown in Figure 3.4(f), copolymerizing with BzDMA significantly improves the water vapor uptake compared with pure PNIPAM. For the copolymers synthesized below the LCST, $15 \%$ is the most suitable amount, because the maximum water vapor uptake could be reached on P(NIPAM-co-15\%BzDMA) which would be selected as the best candidate for coating the PU sponges. 

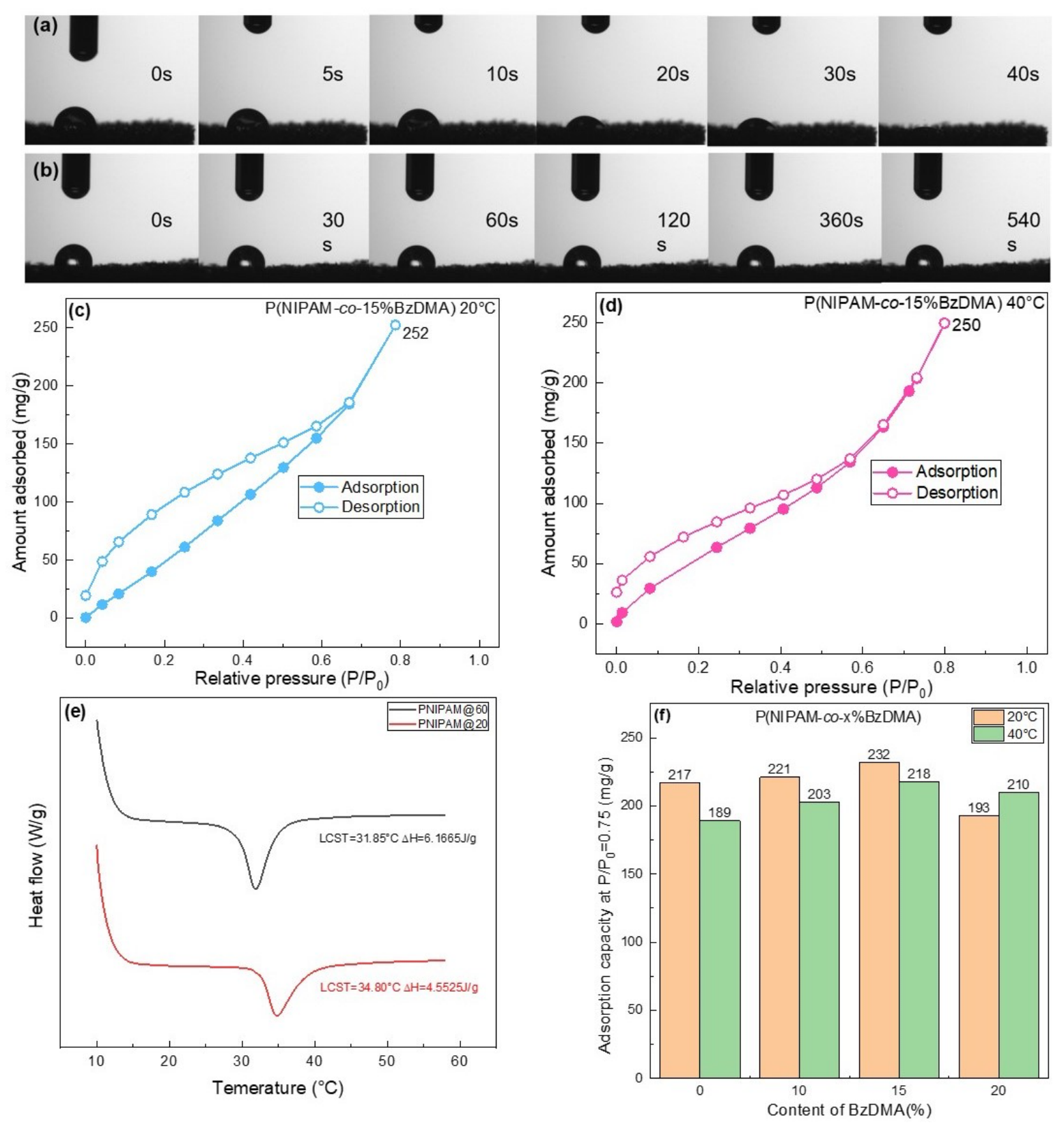

Figure 3.4 Water contact angles of PU-P(NIPAM-co-20\%BzDMA) at (a) $25^{\circ} \mathrm{C}$ and (b) $40^{\circ} \mathrm{C}$ in air, adsorption-desorption isotherms of P(NIPAM-co-15\%BzDMA at (c) $20^{\circ} \mathrm{C}$ and (d) $40^{\circ} \mathrm{C}$, (e) DSC curves of PNIPAM@60, PNIPAM@20, (f) Comparison of the adsorption capacities of $\mathrm{P}(\mathrm{NIPAM}-\mathrm{co}-\mathrm{x} \% \mathrm{BzDMA})$ at a relative pressure of 0.75 . 


\section{Conclusion}

PNIPAM, as a potential polymer for water vapor harvesting due to its hydrophilic-hydrophobic switchable property, two different methods were utilized to further improve its water vapor uptake: synthesis below the LCST and copolymerization with hygroscopic BzDMA, which turned out to be effective in the enhancement of water adsorption capacity. In this way, P(NIPAM-co$15 \%$ BzDMA) was finally determined as the best candidate for PU sponge modification. This work developed an aerogel/sponge composite for capturing atmospheric water from moist air which could be an affordable and energy-efficient way for freshwater collection.

\section{References}

1. Meran, G.; Siehlow, M.; von Hirschhausen, C., The Economics of Water: Rules and Institutions. Springer Nature: 2020.

2. Mekonnen, M. M.; Hoekstra, A. Y. J. S. a., Four billion people facing severe water scarcity. 2016, 2 (2), e1500323.

3. Kim,H.; Yang, S.; Rao, S. R.; Narayanan, S.; Kapustin, E. A.; Furukawa, H.; Umans, A. S.; Yaghi, O. M.; Wang, E. N. J. S., Water harvesting from air with metal-organic frameworks powered by natural sunlight. 2017, 356 (6336), 430-434.

4. Hanikel, N.; Prevot, M. S.; Yaghi, O. M., MOF water harvesters. Nat Nanotechnol 2020, 15 (5), 348-355.

5. Kallenberger, P. A.; Fröba, M., Water harvesting from air with a hygroscopic salt in a hydrogel-derived matrix. Communications Chemistry 2018, 1 (1).

6. Xu, W.; Yaghi, O. M., Metal-Organic Frameworks for Water Harvesting from Air, Anywhere, Anytime. ACS Cent Sci 2020, 6 (8), 1348-1354.

7. Lee, J. J. Harvesting of Water from Fog Using Sponges. 2016.

8. Max, M. D., Atmospheric moisture harvesters. Google Patents: 2007.

9. Loo, S. L.; Vasquez, L.; Paul, U. C.; Campagnolo, L.; Athanassiou, A.; Fragouli, D., Solar-Driven Freshwater Generation from Seawater and Atmospheric Moisture Enabled by a Hydrophilic Photothermal Foam. ACS Appl Mater Interfaces 2020, 12 (9), 10307-10316.

10. Zhao, F.; Zhou, X.; Liu, Y.; Shi, Y.; Dai, Y.; Yu, G. J. A. M., Super Moisture-Absorbent Gels for All-Weather Atmospheric Water Harvesting. 2019, 31 (10), 1806446. 
11. Zhu, H.; Guo,Z.; Liu, W., Biomimetic water-collecting materials inspired by nature. Chem Commun (Camb) 2016, 52 (20), 3863-79.

12. Malik, F. T.; Clement, R. M.; Gethin, D. T.; Krawszik, W.; Parker, A. R., Nature's moisture harvesters: a comparative review. Bioinspiration \& Biomimetics 2014, 9 (3).

13. Yao, X.; Liu, J.; Yang, C.; Yang, X.; Wei, J.; Xia, Y.; Gong, X.; Suo, Z., Hydrogel Paint. Adv Mater 2019, 31 (39), e1903062.

14. Yi, P.; Hu, H.; Sui, W.; Zhang, H.; Lin, Y.; Li, G. J. A. A. P. M., Thermoresponsive Polyurethane Sponges with Temperature-Controlled Superwettability for Oil/Water Separation. 2020, 2 (5), 1764-1772.

15. Yang, H.; Zhu, H.; Hendrix, M. M.; Lousberg, N. J.; de With, G.; Esteves, A. C. C.; Xin, J. H. J. A. M., Temperature-Triggered Collection and Release of Water from Fogs by a Sponge-Like Cotton Fabric. 2013, 25 (8), 1150-1154.

16. Wang, J.; Wang, G.; Wang, W.; Zhang, Z.; Liu, Z.; Hao, Z. J. J. o. M. C. A., Hydrophobic conjugated microporous polymer as a novel adsorbent for removal of volatile organic compounds. 2014, 2 (34), 14028-14037.

17. Fletcher, A. J.; Uygur, Y.; Thomas, K. M. J. T. J. o. P. C. C., Role of surface functional groups in the adsorption kinetics of water vapor on microporous activated carbons. 2007, 111 (23), 8349-8359.

18. Dong, Y.; Zhu, X.; Shi, F.; Nie, J., Surface photo-anchored PNIPAM crosslinked membrane on glass substrate by covalent bonds. Applied Surface Science 2014, 307, 7-12.

19. Azmi, N. S.; Kamaruddin, N. N.; Kassim, S.; Harun, N. A. In Synthesis and characterization of hydrophilic polymer nanoparticles using n-isopropylacrylamide (NIPAM) via emulsion polymerization technique, IOP Conference Series: Materials Science and Engineering, IOP Publishing: 2018; p 012008.

20. Shen, Z.; Bi, J.; Shi, B.; Nguyen, D.; Xian, C. J.; Zhang, H.; Dai, S. J. S. M., Exploring thermal reversible hydrogels for stem cell expansion in three-dimensions. 2012, 8 (27), 7250-7257. 21. Ribeiro, C. A.; Martins, M. V. S.; Bressiani, A. H.; Bressiani, J. C.; Leyva, M. E.; de Queiroz, A. A. A. J. M. S.; C, E., Electrochemical preparation and characterization of PNIPAMHAp scaffolds for bone tissue engineering. 2017, 81, 156-166. 
22. Zhang, M.; Li, Y.; Yang, Q.; Huang, L.; Chen, L.; Xiao, H. J. J. o. M. S., Adsorption of methyl violet using $\mathrm{pH}$-and temperature-sensitive cellulose filament/poly (NIPAM-co-AAc) hybrid hydrogels. 2018, 53 (16), 11837-11854.

23. Guan, Y. L.; Shao, L.; Yao, K. D. J. J. o. A. P. S., A study on correlation between water state and swelling kinetics of chitosan-based hydrogels. 1996, 61 (13), 2325-2335.

24. Smith, S. E. J. J. o.t. A. C. S., The sorption of water vapor by high polymers. 1947, 69 (3), 646-651.

25. Sultan, M.; El-Sharkawy, I. I.; Miyazaki, T.; Saha, B. B.; Koyama, S.; Maruyama, T.; Maeda, S.; Nakamura, T. J. A., Insights of water vapor sorption onto polymer based sorbents. 2015, 21 (3), 205-215. 